\title{
Medical image segmentation using fuzzy c-mean (FCM) and dominant grey levels of image
}

\begin{abstract}
Image segmentation is a critical part of clinical diagnostic tools. Medical images mostly contain noise. Therefore, accurate segmentation of medical images is highly challenging; however, accurate segmentation of these images is very important in correct diagnosis by clinical tools. We proposed a new method for image segmentation based on dominant grey level of image and Fuzzy C-Mean (FCM). In the postulated method, the colour image is converted to grey level image and stationary wavelet is applied to decrease noise; the image is clustered using ordinary FCM, afterwards, clusters with error more than a threshold are divided to two sub clusters. This process continues until there remain no such, erroneous, clusters. The dominant connected component of each cluster is obtained -- if existed. In obtained dominant connected components, the $\mathrm{n}$ biggest connected components are selected. $\mathrm{N}$ is specified based upon considered number of clusters. Averages of grey levels of $\mathrm{n}$ selected components, in grey level image, are considered as Dominant grey levels. Dominant grey levels are used as cluster centres. Eventually, the image is clustered using specified cluster centres. Experimental results are demonstrated to show effectiveness of new method.
\end{abstract}

Keyword: FCM; Dominant grey level; Medical image; Image segmentation 